\title{
Diagnóstico energético eléctrico de un inmueble de oficinas para una dependencia de la administración pública federal
}

Erika Masiel Salinas T.*

Fecha de recepción: 11 de agosto de 2014

Fecha de aprobación: 2 de octubre de 2014

Pp.65-82

\section{RESUMEN}

Se llevó a cabo el diagnóstico energético eléctrico de un inmueble de oficinas perteneciente a una dependencia de la Administración Pública Federal, situado en la zona fronteriza de la ciudad de Matamoros, Estado de Tamaulipas, México. A partir de su funcionamiento, este inmueble comparte instalaciones y facturación con otras dependencias federales. Sin embargo, el pago de la factura eléctrica lo asume por completo la dependencia principal. Los resultados mostraron que el inmueble tuvo un consumo eléctrico facturado de $531866 \mathrm{kWh}$ mientras que el consumo real de la dependencia fue de $234630 \mathrm{kWh}$, lo que representa sólo un $44.1 \%$ de la facturación. Por sus características, este inmueble presentó un Índice de Consumo de Energía Eléctrica (ICEE) de $133 \mathrm{kWh} / \mathrm{m} 2$ que es menor que el Índice de Referencia de $160 \mathrm{kWh} / \mathrm{m} 2$ reportado por CONUEE para inmuebles de la Administración Pública Federal. Después del análisis de la facturación eléctrica y con los resultados del censo de cargas del inmueble para conocer el perfil de consumo eléctrico del mismo, se obtuvieron las potenciales medidas de ahorro económico y de energía; estas son, la adopción de medidas operativas sin inversión, sustitución tecnológica de los sistemas de iluminación, instalación de un banco de capacitores para la compensación de energía reactiva y cambio de transformador y la separación de circuitos para contratar una facturación individual que permita disminuir costos y hacer un seguimiento adecuado de las medidas de eficiencia energética implementadas.

\section{Palabras Clave}

Diagnóstico energético, facturación eléctrica, ahorro de energía, eficiencia de equipos

* Magister en Eficiencia Energética y Ecología Industrial. Universidad de Zaragoza. España. 


\section{Electrical Energy Diagnosis of an Office-Building Used as a Public Administration Building}

\section{Abstract}

Electrical energy diagnosis of an office building belonging to a branch of the Federal Public Administration, located in the border city of Matamoros, Tamaulipas, Mexico, was conducted. The results showed that the house had a power consumption of 531,866 kWh billed while the actual consumption of the unit was $234,630 \mathrm{kWh}$, representing only $44.1 \%$ of consumption described when billing. Due to its characteristics, this property presented an index of electricity consumption (ICEE) of $133 \mathrm{kWh} / \mathrm{m} 2$ which is less than the benchmark set by CONUEE: $160 \mathrm{kWh} / \mathrm{m} 2$ for buildings of the Federal Public Administration. After analyzing the electric billing and, the results of the census to know the power consumption profile of the property, the potential economic saving and energy measures were obtained, which represented operational measures without investment, technological replacement of lighting systems, installation of a capacitor bank for reactive power compensation, change of transformer and circuit separation to hire an individual billing to reduce costs and to allow adequate monitoring of the energy efficiency measures.

\section{KEY WORDS}

Energy diagnosis, electric billing, energy saving, energy consumption, equipment efficiency.

\section{Diagnostic de la consommation d'énergie électrique d'un immeuble de bureaux de l'administration publique fédérale du Mexique}

\section{RÉSUMÉ}

L'article présente un diagnostic de la consommation d'énergie électrique d'un immeuble de bureaux appartenant à une unité de l'administration publique fédérale, située à la frontière de la ville de Matamoros, dans l'état du Tamaulipas, au Mexique. En raison de ses caractéristiques, cet immeuble présente un taux de consommation d'énergie électrique (ICEE) de $133 \mathrm{kWh} / \mathrm{m} 2$, taux inférieur au taux de référence de $160 \mathrm{kWh} / \mathrm{m} 2$ de l'agence CONUEE de l'administration publique fédérale. Après analyse des factures électrique devant servir à mieux connaître le profil de consommation électrique du bâtiment, le potentiel de réduction des coûts et les mesures d'économies d'énergie comme l'adoption de mesures 
opérationnelles sans recours à l'investissement ou la séparation des circuits pour réaliser un suivi des mesures mises en place pour assurer une réduction de la consommation énergétique efficace ont pu être obtenus.

\section{Mots-clés}

Diagnostic énergétique, facturation électrique, économies d'énergie, efficacité des équipements.

\section{Diagnóstico energético elétrico de um prédio de escritórios da Administração Pública Federal}

\section{Resumo}

O artigo apresenta o diagnóstico elétrico de um prédio pertencente a Administração Pública Federal, localizado na cidade fronteiriça de Matamoros, Tamaulipas, México. Devido às suas características, esta propriedade apresentou um índice de Consumo de Energia Elétrica (ICEE) de $133 \mathrm{kWh} / \mathrm{m} 2$, que é menor do que o valor de referência de $160 \mathrm{kWh} / \mathrm{m} 2$ para edifícios relatados por CONUEE para imóveis da Administração Pública Federal. Após a análise da conta de energia elétrica e os resultados do censo da propriedade para determinar o perfil de consumo de energia do mesmo, foi obtido o potencial de medidas de economia de custos e de energia como adoção de medidas operacionais sem investimento ou a separação de circuitos para monitorar adequadamente as medidas de eficiência energética implementadas.

\section{Palavras-chave}

Auditoria energética, faturas de eletricidade, de economia de energia, eficiência de equipamentos. 


\section{Introducción}

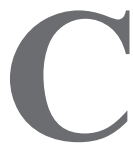
omo parte del Programa de Eficiencia Energética de la Administración Pública Federal, inmuebles de Dependencias Federales que cumplen con ciertas características de arrendamiento, superficie y consumo, se requieren diagnósticos energéticos eléctricos que les permitan identificar el consumo de energía eléctrica, establecer el nivel de eficiencia energética en términos de índices energéticos y conocer los beneficios energéticos y económicos resultantes de establecer medidas de uso eficiente de la energía.

La eficiencia energética se define como la reducción del consumo de energía, manteniendo los mismos servicios energéticos y actividades sin disminuir el confort y la calidad de vida de los usuarios, protegiendo el medio ambiente, asegurando el abastecimiento y fomentando la sostenibilidad de la organización. Por lo anterior, el objetivo principal de este estudio, es realizar un diagnóstico energético eléctrico a través de la realización de un análisis de la facturación eléctrica, con base en la información proporcionada por la dependencia líder; así mismo realizar el censo de cargas del inmueble, presentar el perfil de consumo eléctrico del inmueble, estudiar las ventajas económicas de un cambio de tarifa eléctrica con la compañía suministradora, presentar los potenciales de ahorro de energía en los rubros identificados en el perfil de consumo para, finalmente, elaborar propuestas de ahorro de energía y eficiencia energética y estimar los beneficios económicos y la reducción de emisiones de $\mathrm{CO}_{2}$ equivalente, potenciales.

Este proyecto se llevó a cabo en el inmueble de oficinas de la Dependencia Federal, ubicado en el estado de Tamaulipas, México. La zona en la que se encuentra, limita con la frontera de Estados Unidos de América (Gateway International Bridge, en Brownsville, Texas). Los administradores del inmueble, al solicitar el estudio, refirieron que desde su funcionamiento comparten instalaciones y facturación con otras dependencias federales situadas en el mismo predio. (Figura 1). 
Figura 1. Vista aérea del inmueble

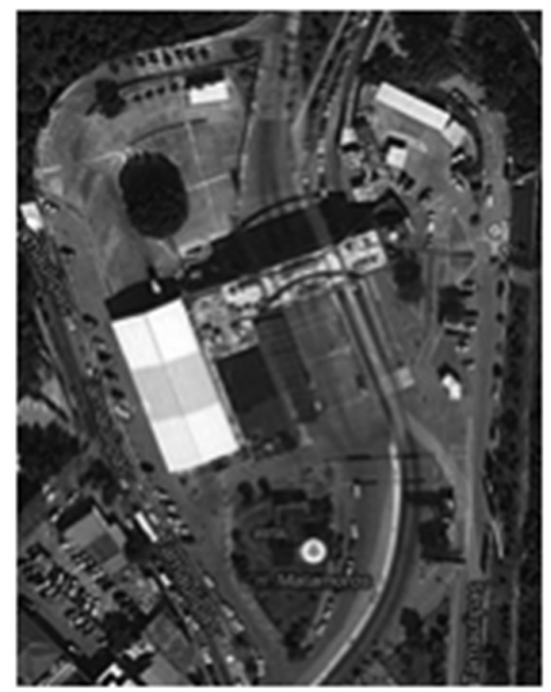

Fuente. Google Maps

En lo que respecta al uso del inmueble, este es de oficinas repartidas en tres edificios de un solo nivel con un área total construida de $4.000 \mathrm{~m}^{2}$. El suministrador del servicio eléctrico es la Comisión Federal de Electricidad (CFE) que lo efectúa en Media Tensión con tarifa de suministro HM. Cuenta con una subestación eléctrica de 500 kVA y una planta de emergencia de $52 \mathrm{~kW}$. Debido a que sus instalaciones utilizan aire acondicionado, le corresponde un ICEE de referencia de $160 \mathrm{kWh} / \mathrm{m}^{2}$-año. 


\section{Metodología}

El diagnóstico energético se realizó con base en la "Guía para elaborar un diagnóstico energético en inmuebles" (Secretaría de Energía, 2013). Se utilizaron los recibos eléctricos proporcionados por la Dependencia Federal para realizar el análisis de la facturación eléctrica. Además, se realizó un censo de cargas del inmueble para conocer el perfil de consumo eléctrico del mismo y se evaluó el cumplimiento con la normatividad aplicable (NOM-007-ENER-2004, NOM-025-STPS-2008 (CONUEE. NOM-007-ENER-2004; CONUEE. NOM-025-STPS-2008). La evaluación de estas normas permitió conocer la eficiencia energética en los sistemas de iluminación, contemplando los niveles de iluminación establecidos por la Secretaría del Trabajo y Previsión Social (STPS), con el objetivo de generar al personal, las condiciones idóneas de trabajo.

Adicionalmente, se realizó un monitoreo de parámetros eléctricos para evaluar la instalación eléctrica, con un analizador de redes y calidad de la energía eléctrica de la marca Circutor, modelo AR5, en el secundario del transformador de distribución, ubicado en la subestación eléctrica que da servicio a las instalaciones de la Dependencia Federal. El equipo se colocó del día 11 al 14 de noviembre, con un total de 72 horas de muestreo, tomando registros a intervalos de cinco minutos durante todo el periodo de monitoreo y almacenando la información obtenida en la memoria interna del mismo.

Una vez detectadas las áreas de mayor consumo eléctrico, se formularon las medidas potenciales de ahorro de energía para los rubros identificados en el perfil de consumo. Se incluyó también un estudio de un cambio de tarifa y una evaluación del factor de potencia. Los resultados se muestran en la siguiente sección. 


\section{Resultados y discusión}

\subsection{Análisis de la facturación}

A partir del análisis de la facturación en un estimado anual, se obtuvo un consumo mensual promedio de $44322 \mathrm{kWh}$ y una demanda facturable promedio de $95 \mathrm{~kW}$. Es asi como se puede observar el consumo mensual y el total de la facturación eléctrica durante un año (Figura 2). Se puede ver que el consumo de energía eléctrica aumenta en los meses de mayo a octubre y disminuye, con respecto a estos, en los meses de noviembre a marzo. Al ser un inmueble que utiliza aire acondicionado, se refleja el aumento en el consumo en las temporadas climáticas.

Figura 2. Consumo de energía eléctrica Vs facturación durante el 2012

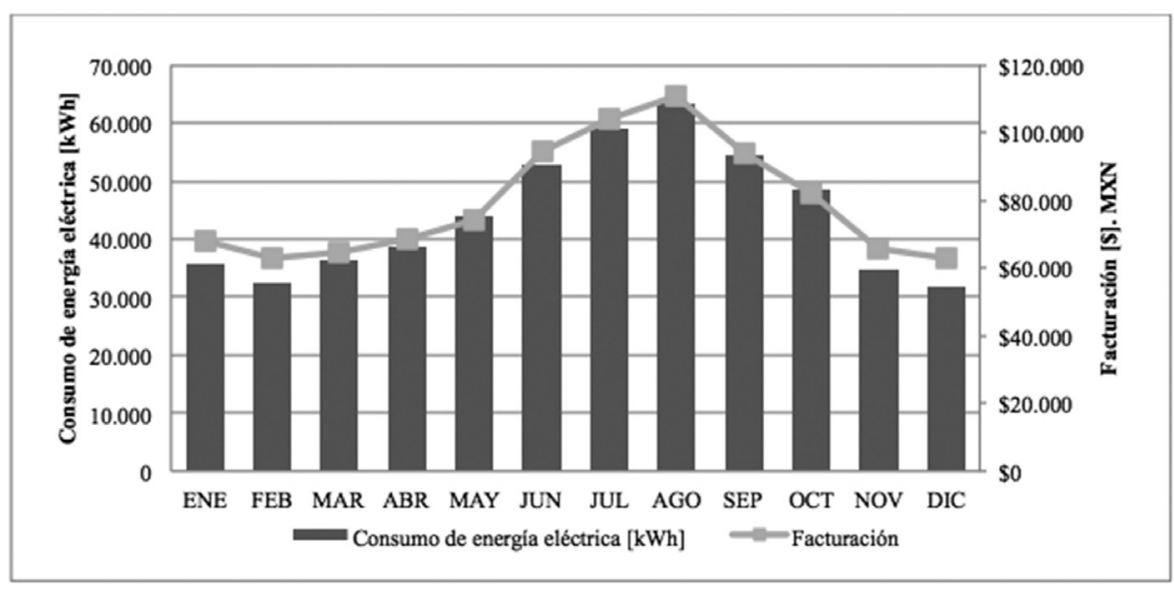

Fuente. Elaboración propia de la autora.

\subsection{Censo de cargas}

\subsubsection{Iluminación}

De la información recabada acerca de la posición de la luminaria, el tipo de gabinete, difusor, lámpara, su potencia y modelo, luces y frecuencia del uso de iluminación en cada área, se observa que la tecnología que predomina son los gabinetes de $4 \times 39 \mathrm{~W}$ (T12), seguida de los 
luminarios de $4 \times 32 \mathrm{~W}$ (T8) y las T8 de $2 \times 32 \mathrm{~W}$. Sin embargo, con base en la distribución de carga, la tecnología predominante son los gabinetes de 4x39 W (T12) con el 58.6\% de la carga, seguida de las lámparas fluorescentes compactas de $150 \mathrm{~W}$ y vapor de sodio de $150 \mathrm{~W}$ con $9.9 \%$ cada una. También se detectó que el $80 \%$ de la carga total instalada es para iluminación interna y el $20 \%$ restante, para iluminación externa, lo que en total representa un consumo energético de $117907 \mathrm{kWh}$ anuales. Para este estudio se evaluaron las normas NOM-007-ENER-2004 y NOM-025-STPS-2008. (Tabla 1).

Tabla 1. Cumplimiento de las normas en iluminación interior

\begin{tabular}{|l|c|c|c|c|}
\hline \multirow{2}{*}{ Inmueble evaluado } & \multicolumn{2}{|c|}{ NOM-025-STPS-2008 } & \multicolumn{2}{c|}{ NOM-007-ENER-2004 } \\
\cline { 2 - 5 } & Cumple & No Cumple & Cumple & No Cumple \\
\hline $\begin{array}{l}\text { Áreas evaluadas en } \\
\text { la norma }\end{array}$ & 3 & 4 & 2 & 6 \\
\hline
\end{tabular}

Fuente. Elaboración propia de la autora.

En cuanto a la NOM-007-ENER-2004, se observa que el $75 \%$ de las áreas evaluadas no cumple con los niveles de eficiencia energética en términos de Densidad de Potencia Eléctrica para Alumbrado (DPEA), que deben cumplir los sistemas de alumbrado de edificios no residenciales. Para conocer si la distribución de la luz es homogénea y adecuada para las condiciones laborales por medio de la medición de luxes, se evaluó la NOM-025-STPS-2008 donde se obtuvo un valor promedio de iluminación por área de trabajo que se comparó con el reportado por la normatividad. Como resultado se tiene que sólo el $44 \%$ de las áreas evaluadas garantiza condiciones idóneas al personal laboral. Se recomienda un cambio de tecnología para mejorar estos parámetros.

\subsubsection{Aire acondicionado}

El sistema de aire acondicionado correspondiente al inmueble consiste en dos equipos de tipo Mini-Split, cada uno de 1.5 T.R. de capacidad, con una antigüedad mayor a cinco años, una potencia medida de $1.98 \mathrm{~kW}$ y un consumo de $8439.55 \mathrm{kWh}$ anuales. Este equipo no cumplió con la NOM023-ENER-2010; sin embargo, al llevar a cabo el estudio de factibilidad y por las características del edificio, una sustitución de tecnología no es económicamente viable. 


\subsubsection{Misceláneos}

En este censo se incluyen los equipos eléctricos utilizados en el área de oficinas que, en conjunto, representan una carga instalada de $9.70 \mathrm{~kW}$ y un consumo anual de $18055.3 \mathrm{kWh}$, c(Tabla 2).

Tabla 2. Resumen del censo de misceláneos

\begin{tabular}{|l|c|c|c|c|}
\hline & $\begin{array}{c}\text { Potencia } \\
{[\mathrm{kW}]}\end{array}$ & $\begin{array}{c}\text { Consumo } \\
{[\mathrm{kWh}]}\end{array}$ & $\begin{array}{c}\text { Carga total } \\
{[\mathrm{kW}]}\end{array}$ & $\begin{array}{c}\text { Consumo } \\
\text { total }[\mathrm{kWh}]\end{array}$ \\
\hline Computo & 0.79 & 17.67 & 0.79 & 17.67 \\
\hline Misceláneo & 8.92 & 32.48 & 8.92 & 32.48 \\
\hline & & Total & 9.70 & 50.15 \\
\hline
\end{tabular}

Fuente. Elaboración propia de la autora.

\subsection{Balance de energía eléctrica}

Después de realizar el censo de cargas y los cálculos correspondientes se realizó el balance de energía eléctrica (Tabla 3).

Tabla 3. Distribución de las cargas eléctricas

\begin{tabular}{|l|c|c|c|}
\hline Carga eléctrica & $\begin{array}{c}\text { Capacidad } \\
\text { Instalada [kW] }\end{array}$ & $\begin{array}{c}\text { Demanda } \\
\text { Máxima [kW] }\end{array}$ & $\begin{array}{c}\text { Consumo } \\
{[\mathrm{kWh} / \mathrm{año}]}\end{array}$ \\
\hline Iluminación interior & 12.22 & 12.2 & 93,715 \\
\hline Iluminación exterior & 3 & 3.0 & 24,192 \\
\hline Aire acondicionado & 2.96 & 3.0 & 8,436 \\
\hline $\begin{array}{l}\text { UMAS (Unidades } \\
\text { Manejadoras de Aire) }\end{array}$ & 0 & 0.0 & 0 \\
\hline Fuerza & 0 & 0.0 & 0 \\
\hline $\begin{array}{l}\text { Equipo de cómputo (Sólo } \\
\text { computadoras y PCs) }\end{array}$ & 0.79 & 0.8 & 6,361 \\
\hline $\begin{array}{l}\text { Contactos (misceláneos) } \\
\text { / otras cargas }\end{array}$ & 8.92 & 5.1 & 11,694 \\
\hline TOTAL & 27.9 & 24.1 & 144,398 \\
\hline
\end{tabular}

Fuente. Elaboración propia de la autora. 
Se identifica que la mayor parte de la energía se utiliza para los sistemas de iluminación interior y exterior, con un $65 \%$ y $17 \%$, respectivamente. Aunque este inmueble comparte instalaciones con otras dependencias federales y, por lo tanto, los consumos tienen el aporte de las mismas, en apartados posteriores se presentarán los potenciales de ahorro de energía para estos sistemas considerando el patrón de consumo del inmueble y su distribución, que resulten rentables y que se someten a consideración de la Dependencia.

\subsection{Monitoreo de parámetros eléctricos}

Como se puede observar, los valores de las mediciones coinciden con las características de la demanda en periodos base, intermedio y punta reportados en la facturación, en la que no hay una diferencia significativa entre uno y otro, incrementando así el costo de la energía (Tabla 4). Los valores de tensión y corriente en las instalaciones eléctricas de la dependencia son adecuados para el funcionamiento de las cargas eléctricas y electrónicas conectadas al sistema.

Tabla 4. Resumen del monitoreo de parámetros eléctricos

\begin{tabular}{|l|l|l|l|l|l|l|}
\hline Concepto & $\begin{array}{c}\text { Tensión } \\
{[\mathrm{V}]}\end{array}$ & $\begin{array}{c}\text { Corriente } \\
{[\mathrm{A}]}\end{array}$ & $\begin{array}{c}\text { Frecuencia } \\
{[\mathrm{Hz}]}\end{array}$ & $\begin{array}{c}\text { Potencia } \\
\text { activa } \\
{[\mathrm{kW}]}\end{array}$ & $\begin{array}{c}\text { Potencia } \\
\text { aparente } \\
{[\mathrm{kVA}]}\end{array}$ & $\begin{array}{c}\text { F. P } \\
{[\%]}\end{array}$ \\
\hline Mínimo & 221.7 & 119.5 & 59.9 & 26.7 & 22.4 & 70 \\
\hline Máximo & 225.2 & 202.6 & 60.02 & 44.9 & 32.0 & 84 \\
\hline Promedio & 222.5 & 156.5 & 60.1 & 34.8 & 16.3 & 77 \\
\hline & & & & & & \\
\hline
\end{tabular}

Fuente. Elaboración propia de la autora.

También se observa un factor de potencia promedio de $77 \%$; este valor es bajo, comparado con el $90 \%$ necesario para bonificación en la facturación eléctrica. Estos valores confirman los porcentajes de recargo aplicados por la Comisión Federal de Electricidad en la facturación. 


\subsection{Estudio de cambio de tarifa y propuesta de ahorro económico}

El inmueble recibe un suministro en media tensión, entre los 1,000 y 35,000 V. Para este servicio, la CFE presenta las siguientes tarifas:

- OM: se aplica a los servicios que destinen la energía a cualquier uso, suministrados en media tensión, con una demanda menor a $100 \mathrm{~kW}$

- HM: se aplica a los servicios que destinen la energía a cualquier uso, suministrados en media tensión, con una demanda de $100 \mathrm{~kW}$ o más.

- H-MC: se aplica a los servicios que destinen la energía a cualquier uso, suministrados en media tensión en las regiones de Baja California y Noroeste, con una demanda de 100 kW o más, y que por las características de utilización de su demanda soliciten inscribirse en este servicio, el cual tendrá vigencia mínima de un año.

De acuerdo con el historial resultante de los recibos eléctricos proporcionados por la Dependencia, la demanda máxima del inmueble es mayor a $100 \mathrm{~kW}$, por lo que la tarifa OM no cubriría el total; en cambio, la H-M y $\mathrm{H}-\mathrm{MC}$ suministran lo requerido. Considerando que la tarifa $\mathrm{H}-\mathrm{MC}$ sólo es aplicable para la región de Baja California y Noroeste, la tarifa H-M resulta ser la más adecuada y no se necesita realizar el cambio a otro tipo de tarifa.

\subsection{Evaluación del factor de potencia y propuesta de ahorro económico}

Se observa, de acuerdo con la facturación a lo largo del año, un factor de potencia inferior al $90 \%$, lo que representa recargos en el monto mensual de la facturación de hasta $\$ 46205.00$ anuales (Tabla 5). 
Tabla 5. Pérdidas por recargos registradas en la facturación

\begin{tabular}{|l|l|c|c|}
\hline Mes & F. P. [\%] & Recargos [\%] & Pérdidas por recargos \\
\hline Enero & 78.27 & 8.99 & $\$ 6,144.65$ \\
\hline Febrero & 77.61 & 9.58 & $\$ 5,634.78$ \\
\hline Marzo & 79.73 & 7.73 & ND \\
\hline Abril & 82.82 & 5.20 & $\$ 5,204.62$ \\
\hline Mayo & 83.92 & 4.35 & $\$ 3,682.35$ \\
\hline Junio & 83.01 & 5.05 & $\$ 4,121.38$ \\
\hline Julio & 84.30 & 4.06 & $\$ 2,753.98$ \\
\hline Agosto & 82.47 & 5.48 & \$4,148.70 \\
\hline Septiembre & 82.32 & 5.60 & $\mathrm{ND}$ \\
\hline Octubre & 80.78 & 6.85 & $\$ 4,952.96$ \\
\hline Noviembre & 76.64 & 10.46 & $\$ 5,076.55$ \\
\hline Diciembre & 74.80 & 12.19 & \\
\hline & Total & $\$ 46,205.83$ & \\
\hline
\end{tabular}

Fuente. Elaboración propia de la autora.

La compensación de la energía reactiva que corrige un bajo factor de potencia puede realizarse a través de la instalación de un banco de capacitores. De acuerdo con las características técnicas del inmueble, se propone la instalación en el sistema eléctrico de un banco de capacitores automático de 50 kVA para 240 Volts, NEMA1 de 5 o 7 u 11 pasos por kVAr con interruptor termomagnético como protección. Este equipo tiene un costo de inversión aproximado de $\$ 199.350 .00$ pesos. Considerando los recargos aplicados a la facturación de 2012 se tiene un ahorro por eliminación de recargos por bajo factor de potencia de $\$ 46205.83$, lo que en un estudio de factibilidad financiera, utilizando un TREMA de $12 \%$ y un periodo de evaluación de diez años, resulta en los siguientes parámetros de evaluación económica (Tabla 6).

Tabla 6. Parámetros de evaluación económica

\begin{tabular}{|l|l|}
\hline \multicolumn{2}{|c|}{ Evaluación Económica } \\
\hline VPN & $\$ 61,723$ \\
\hline B/C & 1.31 \\
\hline TIR & $19 \%$ \\
\hline PR Simple [años] & 4.3 \\
\hline
\end{tabular}

Fuente. Elaboración propia de la autora. 
Esta propuesta se considera económicamente viable debido a los resultados ampliamente positivos de cada uno de los métodos y al tiempo de recuperación de la inversión que se reflejarán en el monto mensual de facturación, una vez que se pase del esquema de penalización al de bonificación. Además de los ahorros conseguidos, se disminuyen las pérdidas de energía en motores, cables y transformadores. Sin embargo, se deja a consideración de la dependencia la implementación de este proyecto de acuerdo con criterios propios y evaluaciones que para sus intereses convenga.

\section{Propuestas de ahorro económico y de energía}

\subsection{Sustitución tecnológica en iluminación}

Se propone la sustitución de tres tecnologías de iluminación, como se detalla a continuación:

\subsection{1 Área común del inmueble}

Sustitución por 40 lámparas de vapor de sodio a alta presión de 50 $\mathrm{W}$, que son más eficientes tanto por sus características técnicas como de consumo. Utilizando el mismo tiempo de operación de 8.064 horas

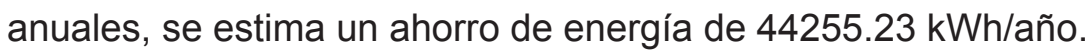

\subsection{2 Área exterior}

Zona de revisión de autobuses: sustitución por siete lámparas de vapor de sodio a alta presión con una potencia de $100 \mathrm{~W}$, que proporciona un flujo luminoso mayor en comparación con una lámpara fluorescente compacta. La sustitución por esta tecnología, bajo las mismas condiciones de uso, representaría un ahorro de energía anual de $1411.2 \mathrm{kWh}$. 
Si se considera un costo de inversión aproximado de $\$ 58.950$, que comprende la instalación y compra de las lámparas, una TREMA del 12\%, un precio de la energía eléctrica de $1.4 \$ / \mathrm{kWh}$ y un periodo de evaluación de cinco años, se obtienen los siguientes parámetros económicos que resultan viables (Tabla 7 ).

Tabla 7. Parámetros de evaluación económica

\begin{tabular}{|l|l|}
\hline \multicolumn{2}{|c|}{ Evaluación Económica } \\
\hline VPN & $\$ 178636$ \\
\hline B/C & 4.03 \\
\hline TIR & $109 \%$ \\
\hline PR Simple [años] & 0.9 \\
\hline
\end{tabular}

Con esta medida se calcula una reducción anual de aproximadamente 23.2 [tCO2eq/año] dando un total de 116 [tCO2eq] a lo largo de los cinco años de la evaluación.

\subsubsection{Cambio de transformador}

Se propone el cambio del transformador de 500 kVA debido a que el inmueble presentó una demanda evaluada máxima de 128 kW; esto es sólo el $26 \%$ de utilización de la subestación. Además, al ser un transformador antiguo, requiere para su funcionamiento un consumo de energía eléctrica de alrededor de $2 \%$ de su capacidad, por lo que al tener una capacidad instalada que excede a la que realmente se necesita, presenta un consumo eléctrico que se traduce en pérdidas energéticas y económicas para el consumidor. Así, se recomienda un transformador de $225 \mathrm{kVA}, 13.2 \mathrm{kV} / 220$ - $127 \mathrm{~V}$ con un porcentaje de 1\% para energizarse,

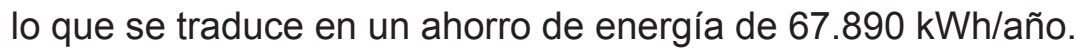

Considerando los valores de consumo eléctrico, se calcularon las emisiones resultantes, evitadas por este cambio, en un aproximado de 33.46 [ $\mathrm{tCO}_{2}$ eq/año] dando un total de 334.63 [ $\mathrm{tCO}_{2}$ eq] durante los diez años de evaluación. 


\subsection{Separación de circuitos y facturación individual}

La facturación del inmueble refleja las demandas y consumo de varias dependencias ajenas a la dependencia líder. Al evaluar una separación de contrato utilizando el valor máximo de la demanda facturable en los horarios intermedio y punta y considerando que las mediciones mostraron que la dependencia líder sólo consume una tercera parte de lo que aparece en la facturación, a continuación se observan los resultados por separado debido a que en el mes de horario de verano se incrementa el consumo y la demanda por los equipos de aire acondicionado (Tabla 8).

Tabla 8. Costo de la facturación aplicando una separación de circuitos

\begin{tabular}{|l|l|}
\hline Periodo & Costo \\
\hline 6 meses fuera de verano & \\
\hline $136,398.252$ & \\
\hline 6 meses en verano & $215,064.974$ \\
\hline Total anual & $351,463.226$ \\
\hline
\end{tabular}

Fuente. Elaboración propia de la autora.

Con lo anterior, se observa un costo de facturación anual de $\$ 351,463$ pesos. Al considerar que actualmente se tiene una facturación anual de $\$ 951,922$ pesos se tendría un ahorro de $\$ 600,459$ pesos, por lo que se recomienda se haga un contrato nuevo ante la CFE en el que solo se facture lo consumido.

\subsection{Medidas operativas sin inversión}

Se recomienda implementar una campaña de concientización de ahorro de energía con el objetivo de evitar que los equipos de cómputo se queden encendidos durante horas posteriores a la jornada laboral, esto es de las 18 horas a las ocho horas del día siguiente, así como, apagar las luces en áreas que no se ocupan por ser lugares de poco acceso de personal. Por ejemplo, bodegas de archivos, área de la subestación y oficinas que no se estén ocupando. Finalmente, desconectar equipos con energía en espera (hornos de microondas, cafeteras, televisiones, despachadores de agua por las noches, y todos aquellos equipos que tengan un consumo por energía en espera o stand-by). 


\section{Conclusiones}

Se realizó el diagnóstico energético eléctrico en un inmueble de oficinas de una dependencia federal. Según los resultados del censo de carga y el consumo mensual, se concluye que estas sólo representan un $44.1 \%$ de la facturación, lo que indica que aproximadamente un $60 \%$ se debe al consumo de otras dependencias ajenas a la dependencia líder. Tomando en cuenta el consumo anual y el área del inmueble se obtiene un ICEE real de $133 \mathrm{kWh}$. Sin embargo, con la adopción de las medidas de ahorro de energía propuestas, el ICEE disminuiría a $105 \mathrm{kWh}$, estimando una reducción de emisiones de 76 ton $\mathrm{CO}_{2}$ eq con respecto a la situación actual.

En cuanto al programa de CONUEE para la promoción de la eficiencia energética esta deberá establecer mecanismos para monitorear los resultados de las Dependencias Federales pues se corre el riesgo de una falta de continuidad en los reglamentos de política sobre eficiencia energética en el país. Se espera que en lo sucesivo y gracias al apoyo que en México se está dando a la promoción de la eficiencia energética se puedan mejorar e introducir programas para monitorear los resultados de los indicadores de desempeño en eficiencia energética utilizando los resultados de estudios similares al que se ha llevado a cabo.

\section{Agradecimientos}

La autora de este artículo agradece a la Fundación Carolina por financiar la estancia en España y los estudios del Máster en Eficiencia Energética y Ecología Industrial. Al comité evaluador de becarios de la Universidad de Zaragoza y CIRCE. A la doctora Tanya Moreno Coronado, a los profesores del CIRCE y en especial, a don Antonio Valero y doña Alicia Valero Delgado por la pasión con la que transmiten sus ideas y la semilla que siembran en quienes los escuchan. 


\section{Referencias bibliográficas}

CONUEE. NOM-007-ENER-2004 Eficiencia energética en sistemas de alumbrado en edificios no residenciales. Recuperado de: http://www. conuee.gob.mx/wb/CONAE/CONA_1002_nom_publicadas_vigen

CONUEE. NOM-025-STPS-2008: Condiciones de iluminación en los centros de trabajo. Recuperado de: http://www.conuee.gob.mx/wb/ CONAE/CONA_1002_nom_publicadas_vigen

CONUEE. NOM-023-ENER-2010 Eficiencia energética en acondicionadores de aire tipo dividido, descarga libre y sin conductos de aire. Límites, método de prueba y etiquetado. Recuperado de: http://www. conuee.gob.mx/wb/CONAE/CONA_1002_nom_publicadas_vig

Secretaría de Energía, 2013.. CONUEE, Guía para elaborar un diagnóstico energético en inmuebles. 
